\title{
PLANEJAMENTO, ORDENAMENTO E GESTÃO INTEGRADA ECOLOGIA DE PAISAGEM E ANÁLISE INTEGRADORA DA PAISAGEM DA SUB-BACIA DO RIO DOS PATOS
}

\author{
Grenda Costa Cordeiro Alves - grenda_cordeiro@ hotmail.com \\ Universidade Tecnológica Federal do Paraná.
}

Paulo Vinícius Faleiro de Almeida - paulinhopvfa@ @otmail.com Universidade Tecnológica Federal do Paraná.

Vanessa Laura dos Santos - vanessalaurasantos@ hotmail.com Universidade Tecnológica Federal do Paraná.

\begin{abstract}
Aline dos Santos Ferreira - alinesantossf@hotmail.com
Universidade Tecnológica Federal do Paraná.
\end{abstract}

Edivando Vitor do Couto - edivandocouto@ gmail.com Universidade Tecnológica Federal do Paraná.

\begin{abstract}
RESUMO: Neste trabalho foi realizado o mapeamento da Sub-Bacia do Rio dos Patos, sendo esta uma parcela da Bacia do Rio Ivaí, abrangendo os municípios de Prudentópolis e Irati, ambos situados no estado do Paraná. O mapeamento foi realizado levando em conta os dados obtidos pela Agência Nacional das Águas (ANA), já delimitadas para o Sistema de Informação Geográfico (SIG, QGIS, versão 2.10.1). A ferramenta complementar Landscape Ecology Statistics - LecoS, foi utilizado para o cálculo das métricas quanto às classes ou paisagens. Este estudo se torna muito importante, uma vez que com a análise e interpretação das métricas disponibilizadas é possível propor uma integração entre os fragmentos florestais, bem como propostas para o manejo de conservação.
\end{abstract}

Palavras-chave: Fragmentos Florestais; Métricas; Sub-Bacia dos Patos.

\section{INTRODUÇÃO}

O ambiente natural é o melhor abrigo para espécies nativas e/ou migratórias (LOUZADA et al., 2010). No entanto, com a intensa expansão agrícola e urbana, a vegetação brasileira sofre com a redução dos sistemas naturais tornando-os fragmentos florestais isolados de sua paisagem original e criando barreiras que diminuem a interação de fauna e flora nos remanescentes do local, ocasionando mudanças nas relações ecológicas.

A ecologia da Paisagem é a combinação do estudo do geógrafo com o ecologista para interpretar as relações funcionais de uma determinada área descrevendo tudo o que há nele, desde pequenos organismos até a Terra no geral. O parâmetro mais importante da ecologia da 

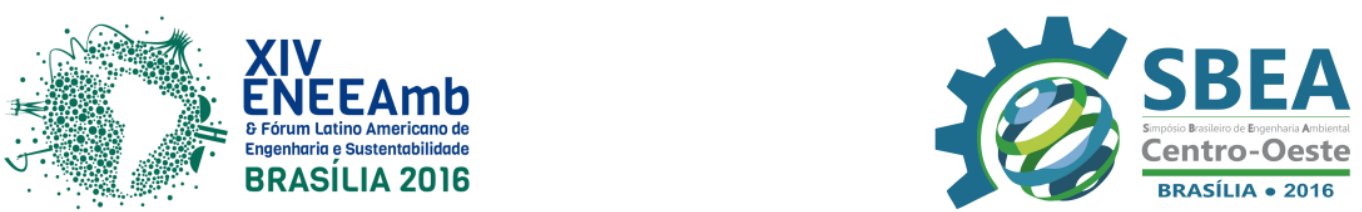

paisagem é a relação entre estrutura da paisagem e os processos ecológicos (BATISTA, 2014).

Para a interpretação da medição e análise dos parâmetros é necessário o uso das métricas de paisagem, as quais foram calculadas através do LECOS®. Essas métricas são utilizadas como uma ferramenta de auxílio para a interpretação do ambiente, ajudando a descrever a estrutura espacial e a classe das manchas (BATISTA input Botequilha Leitão et al. 2006).

Portanto, o presente trabalho tem por objetivo efetuar uma análise integradora dos fragmentos de paisagem na sub bacia do Rio dos Patos, pertencente à bacia do rio Ivaí Paraná, podendo utilizar esses resultados para propor corredores ecológicos ou algum outro tipo de manejo para o local.

\section{1 Área de estudo}

O presente estudo foi realizado sobre a Sub Bacia do Rio dos Patos que compreende os municípios de Irati e Prudentópolis no Estado do Paraná (Figura 1). De acordo com a Secretaria de Estado do Meio Ambiente e Recursos Hídricos - SEMA (2010) a área está situada na bacia do Rio Ivaí (Figura 2), compreendendo o alto do Ivaí, nas coordenadas geográficas Longitude entre $51^{\circ} 10^{\prime}$ e $51^{\circ} 50^{\prime} \mathrm{O}$ e latitude entre $25^{\circ} 00^{\prime}$ e $25^{\circ} 30^{\prime} \mathrm{S}$.

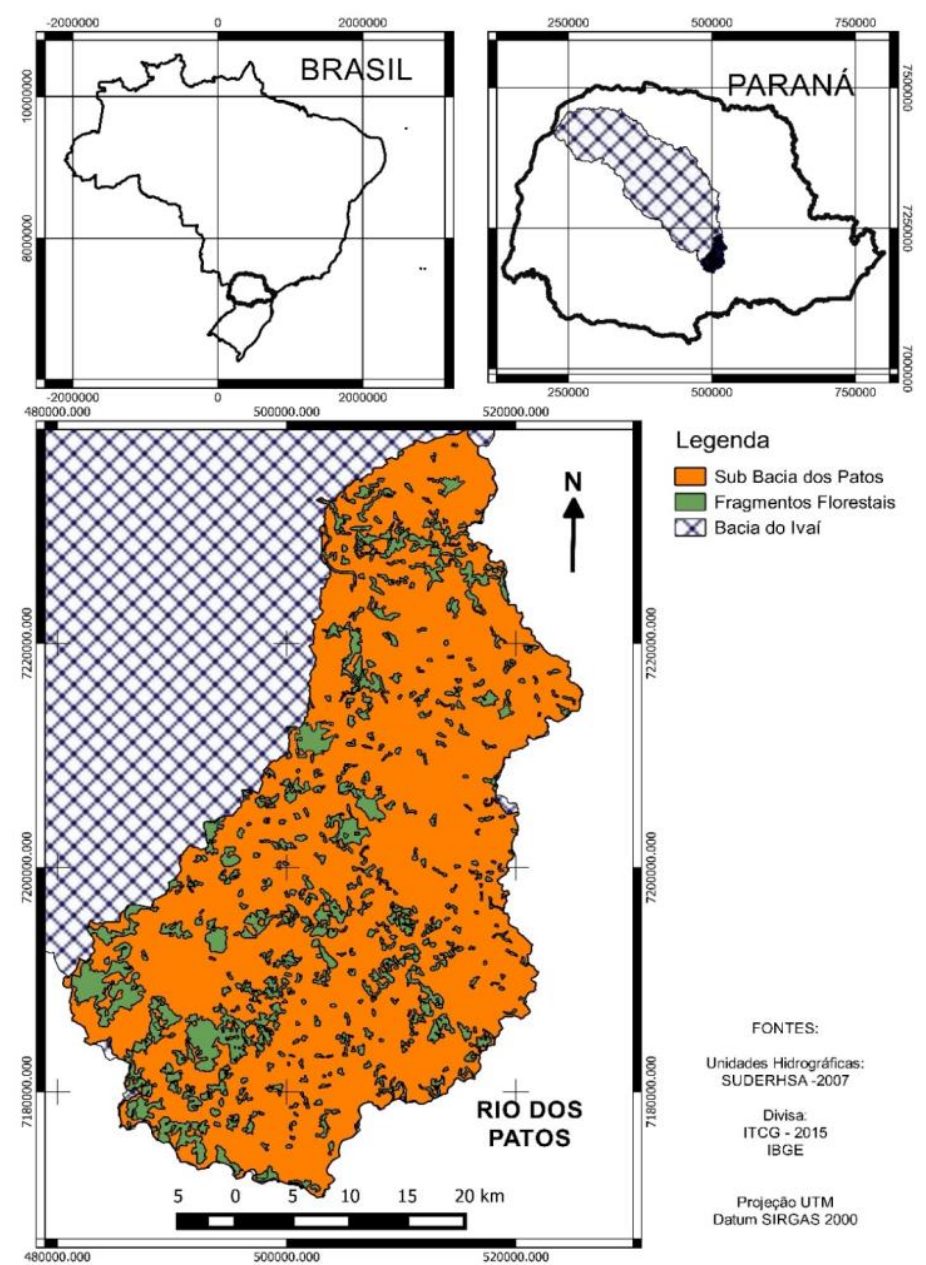

Figura 1: Mapa de localização da sub-bacia do Rio dos Patos. 

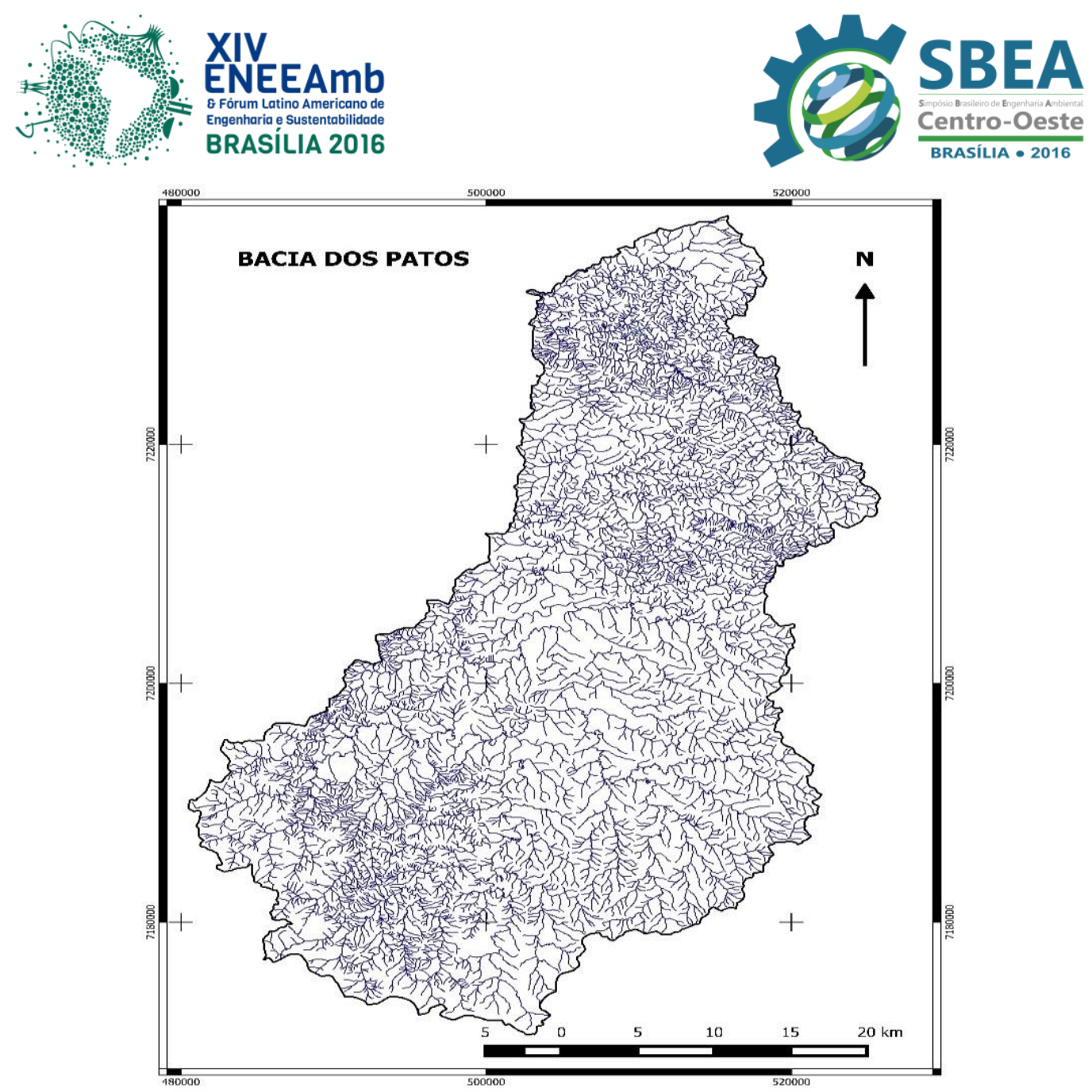

Figura 2: Mapa de hidrografia Rio dos Patos.

\subsection{Formações florestais}

De acordo com o mapa de fitogeografia do ITCG (2009), a vegetação presente na sub bacia hidrográfica do Rio dos Patos é a Floresta Ombrófila Mista Montana, formação de grande abundância na região (IBGE, 1993) que apresenta-se em face da altitude e latitude do planalto meridional, segundo Veloso et al. (1991).

A porção florestal que recobre está área possui características tipicamente subtropicais com espécies de pinheiros, entre eles a Araucária angustifólia, pinheiro símbolo do estado do Paraná (ITCG, 2009). As principais atividades econômicas que prevalecem na região são a agricultura e a pecuária.

\subsection{Descrição geológica e geomorfológica}

A área estudada possui as características geológicas de maioria argilito, siltito, arenito fino e muito fino, em outra parcela apresenta rochas aelito e arenito, e uma pequena área de basalto e basalto tholeítico (MINEROPAR, 2001).

A Unidade morfoestrutural da area estudada é a bacia sedimentar do Paraná na unidade morfoescultural do segundo planalto paranaense com as sub-unidades morfoesculturais do Planalto de Irati, Planaltos residuais de formações Serra Geral e Planalto de Prundentópolis (MINEROPAR, 2006).

Segundo Embrapa Solos (2006) não há predomínio de apenas um tipo de solo. A região apresenta Neossolos litólicos eutróficos, Argissolos vermelho - amarelos distróficos, Latossolos vermelhos eutroférricos e Latossolos Brunos distróficos. 

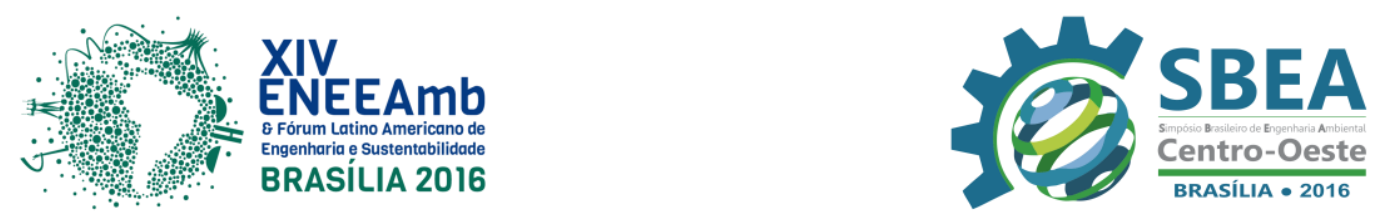

\subsection{Clima}

Wladimir Peter Köppen apresenta o clima da região como $\mathrm{Cfb}$ (clima temperado úmido com verão temperado). Utilizando desse sistema, e baseando na vegetação e temperatura, o Instituto Agronômico do Paraná (IAPAR) classificou o clima do local estudado como subtropical, caracterizado por invernos com temperaturas médias inferiores a $18^{\circ} \mathrm{C}$ e verão frescos com temperaturas médias

\section{MATERIAIS E MÉTODOS}

O banco de dados da bacia hidrográfica do Ivaí, bem como sua subdivisão - Rio dos Patos, foram disponibilizadas pela Agência Nacional das Águas (ANA) e delimitados para o Sistema de Informação Geográfico (SIG) QGIS® versão 2.10.1. A ferramenta complementar Landscape Ecology Statistics - LecoS foi utilizado para o cálculo das métricas quanto às classes ou paisagens.

\subsection{Métricas de área e borda}

A área apresenta uma importante base para o cálculo de várias métricas de manchas, classe e paisagem, possuindo assim grande influência em inúmeros processos ecológicos. Existe, por exemplo, evidências de que o tamanho de determinadas manchas pode influenciar na riqueza de aves e na ocorrência de algumas espécies no local (ROBBINS et. al.,1989). Grande parte das espécies possuem dependências de uma área mínima para sobreviverem, sendo chamadas de espécies sensíveis à área.

- Área de cada classe na camada $\left(\mathbf{m}^{2}\right)$ : área total da paisagem ocupada por uma classe, manchas maiores tem maior probabilidade de conter espécies de interior, dependendo da sua configuração (MCGARIGAL \& MARKS et.al. 1995)

- Proporção da área da classe (\%): proporção da paisagem composta por uma determinada classe, permitindo quantificar a extensão relativa que cada classe ocupada na paisagem (MCGARIGAL \& MARKS 1995; BOTEQUILHA LEITÃO et al. 2006).

- Comprimento de borda (m): somatório de todos os comprimentos de todos os segmentos de borda contidos em uma área, métrica derivada do perímetro, sendo o total de borda (MCGARIGAL \& MARKS, 1995).

- Densidade de borda $\left(\mathbf{m} / \mathbf{m}^{2}\right)$ : calculado a partir do comprimento total de borda da paisagem pela área total da paisagem.

- Área do maior e menor fragmento $\left(\mathbf{m}^{2}\right)$ : porção da paisagem ocupada pela maior/menor mancha, permite observar se a paisagem é dominada por uma só mancha e assim avaliar a sua homogeneidade (MCGARIGAL \& MARKS, 1995).

- Média da área dos fragmentos $\left(\mathbf{m}^{2}\right)$ : tamanho médio das manchas de uma classe ou paisagem, permitindo perceber como se comporta o tamanho das manchas na classe ou paisagem (MCGARIGAL \& MARKS, 1995).

- Mediana da área dos fragmentos $\left(\mathbf{m}^{2}\right)$ : representa $50 \%$ do tamanho médio das manchas de uma classe ou paisagem, permitindo perceber como se comporta o tamanho das manchas na classe ou paisagem. 

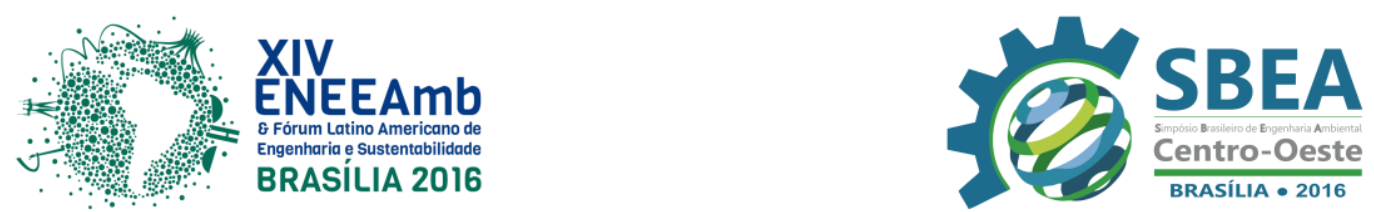

\subsection{Métricas de fragmentação}

A fragmentação de um habitat pode afetar uma espécie ou um processo ecológico, tendo em vista que, populações de uma espécie pode se isolar nos fragmentos e conectar-se eventualmente com outras, através de poucos indivíduos que se movem para outros fragmentos. (GILPIN \& HANSKI et al., 1991). Quanto maior a distância entre as manchas, maior será a fragmentação do ambiente, tendo menos áreas continuas de vegetação, menores serão os espaços para a reprodução de fauna e flora, reduzindo populações das mesmas. As métricas de fragmentação são muito utilizadas na análise de adequação da paisagem como habitats para espécies de fauna, relacionadas com análises binárias de presença ou ausência de um determinado tipo de habitat essencial para a espécie em causa (HAILA et al. 1987; DUNNING et al., 1992; GUSTAFSON et al., 1994).

- Número de manchas: representa o número total de manchas em uma paisagem, quanto maior o número de manchas maior será a fragmentação do local.

- Densidade de manchas: número total de manchas numa classe ou paisagem por unidade de área.

\section{RESULTADOS E DISCUSSÕES}

A classe de área definida como matriz (Sub Bacia dos Patos) obteve $1.324 .880 .000 \mathrm{~m}^{2} \mathrm{de}$ cobertura de terra, abrangendo $84,48 \%$ da paisagem da sub-bacia. Os 403 fragmentos florestais totalizam $225.000 .000 \mathrm{~m}^{2}$ de cobertura da terra, sendo responsável por $14,42 \%$ da proporção da paisagem, analisados na Figura 3:

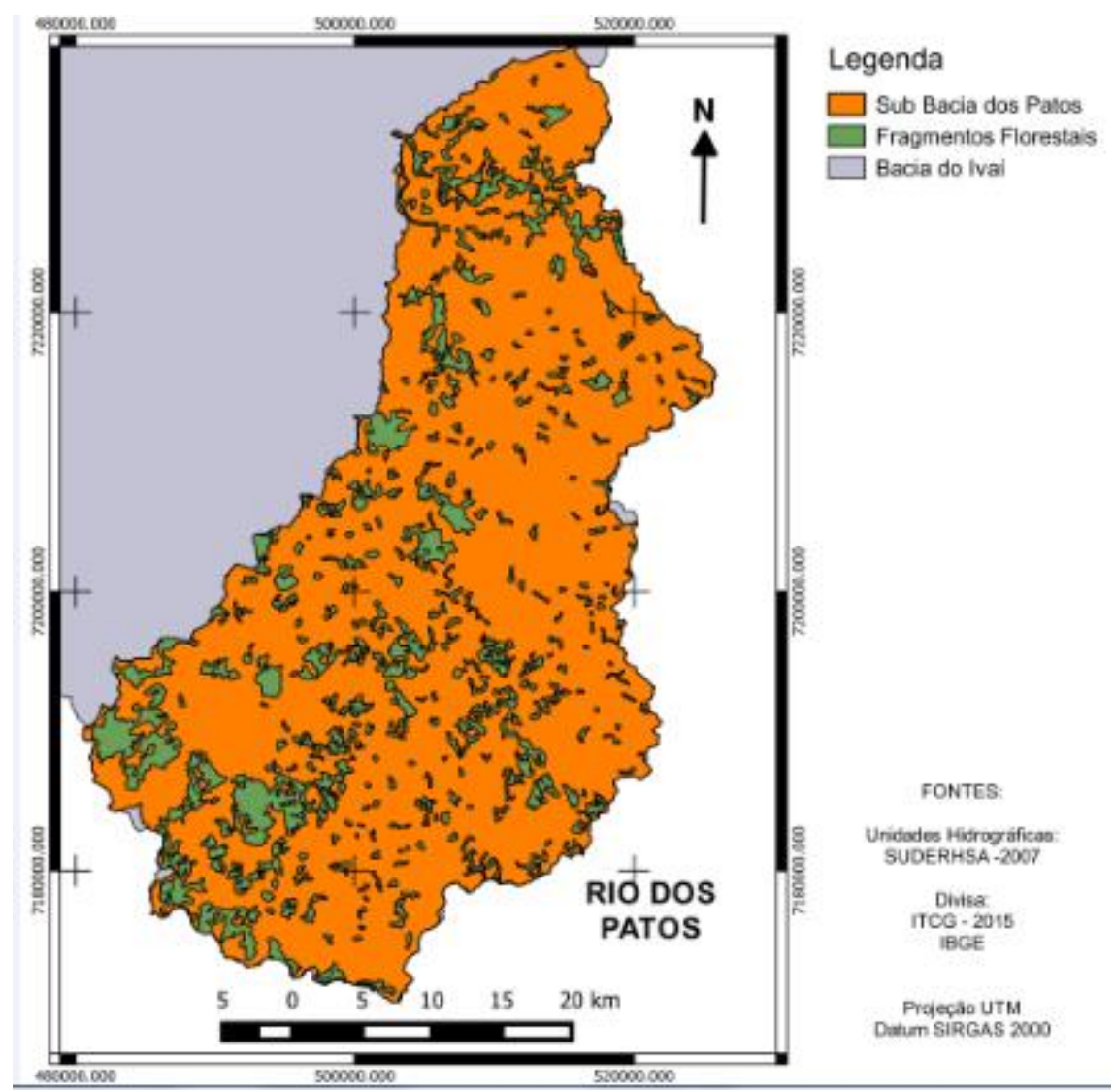

Figura 3: Sub-Bacia do Rio dos Patos com área de matriz e fragmentos florestais. 

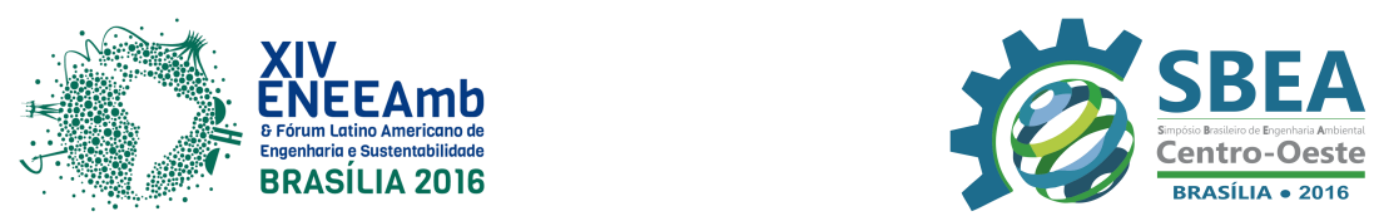

Em relação ao comprimento de borda os fragmentos florestais apresentaram um valor de 1.779.200 metros, gerando para cada fragmento, em média, 4.414 metros de comprimento de borda, se esse valor for alto e simultaneamente o formato do fragmento for alongado, haverá o efeito negativo no fragmento. Observou-se ainda, com o resultado de densidade de borda de $0.00114795 \mathrm{~m} / \mathrm{m}^{2}$, que quanto maior o número de fragmentos de uma determinada classe, maior será o índice de densidade de borda, e consequentemente, haverá elevada fragmentação da paisagem (MCGARIGAL et al., 2012). Assim, os fragmentos maiores estão melhores conservados por possuírem menor densidade de borda, logo, menos sujeitos aos chamados "efeitos de borda".

Para a análise da complexidade do fragmento em relação à sua forma, foi utilizado o índice de Patton, o qual considera que quanto maior o valor, maior será a complexidade do fragmento, com maior ocorrência de microambientes, e, portanto, maior efeito de borda (SOUZA et al., 2008). O maior fragmento apresentou $21.080 .000 \mathrm{~m}^{2}$ e índice de Patton de aproximadamente 6, já o menor, amostrou uma área de $40.000 \mathrm{~m}^{2} \mathrm{e}$ índice de Patton próximo a 3 .

A área em estudo apresentou, também, $2.60 \times 10^{-7}$ manchas de fragmentos por $\mathrm{m}^{2} \mathrm{de}$ área e um índice de densidade de 0.26 , o qual representa a quantidade de manchas para cada 100 ha. Segundo Guimarães \& Lobão (2013), o índice de densidade de borda varia de 0 a 1 , sendo que quanto mais próximo de zero maior é a heterogeneidade ecológica do ambiente. Logo, é possível observar que a área em estudo apresenta elevado grau de heterogeneidade na paisagem, prejudicando a recuperação do bioma.

Os valores de média e mediana para os fragmentos foram, respectivamente, de $558.312,65 \mathrm{~m}^{2}$ e $160.000 \mathrm{~m}^{2}$, podendo perceber a presença de muitos fragmentos pequenos juntamente com outros de áreas consideravelmente maior.

\section{CONCLUSÃO}

$\mathrm{Na}$ área de estudo encontrou-se uma predominância de fragmentos pequenos e bem espaçados, podendo resultar em redução da biodiversidade e dos processos ecológicos. Para sua melhoria seria necessário, primeiramente, a recuperação das margens dos rios com espécies principalmente nativas, tendo em vista a grande quantidade de rios na região, e em seguida, a criação de propostas de corredores ecológicos com o objetivo de aumentar as áreas florestais e a biodiversidade do local.

Considerando-se que as principais atividades econômicas são a agricultura e a pecuária, uma técnica viável para a regeneração e conservação seria a integração lavourapecuária-floresta, que além dos benefícios ambientais, garante vantagens para o produtor, tanto na preservação do solo de sua propriedade, quanto economicamente.

\section{REFERÊNCIAS}

AGENCIA NACIONAL DAS ÁGUAS. Bases de Dados Georreferenciadas. Disponível em: http://www.ana.gov.br/bibliotecavirtual/solicitacaoBaseDados.asp. Acesso em: 20 de Novembro de 2015.

BATISTA, M. T. F. Modelação geográfica em processos de caracterização e avaliação da paisagem numa perspetiva transfronteiriça. Universidade de Évora. Évora, jun. 2014. 

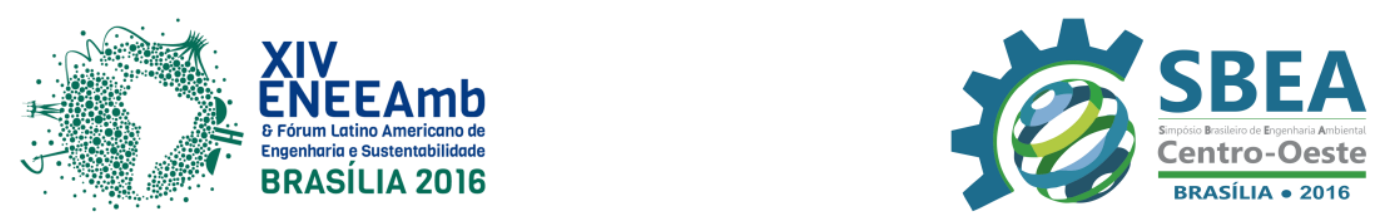

BOTEQUILHA LEITÃO A.; J. MILLER, J.; AHERN \& MCGARIGAL, K.; Measuring Landscapes: A Planner's Handbook. Washington DC, Island Press., 2006.

DUNNING J.B.; DANIELSON, B.J. \& PULLIAM, H.R. Ecological processes that affect populations in complex landscapes. Oikos, 1992. p.169-175.

EMBRAPA SOLOS, 2006. Levantamento de reconhecimento dos solos do estado do Paraná. Disponível em:

http://www.infoteca.cnptia.embrapa.br/bitstream/doc/339505/12/MI505.pdf. Acesso: 15 nov. 2015.

GILPIN, M. E. \& HANSKI, I. (EDS). Metapopulation Dynamics: Empirical and Theoretical Investigations. Academic Press, San Diego, 1991. p. 336.

GUIMARÃES, T. L. B.; LOBÃO, J. S. B. Sistemas de Informações Geográficas para análise de fragmentos de vegetação no Polo de Jeremoabo. Anais XVI Simpósio Brasileiro de Sensoriamento Remoto, Foz do Iguaçu, PR, Brasil, abril de 2013. Disponível em: http://www.dsr.inpe.br/sbsr2013/files/p0927.pdf

GUSTAFSON, E.J. \& PARKER, G.R. Relationships between landcover proportion and indices of landscape spatial pattern. Landscape Ecology, 1992. p. 101-110.

HAILA, Y.; HANSKI, I.K. \& RAIVIO, S. Breeding bird distribution in fragmented coniferous taiga in southern Finland. Ornis Fennica, 1987. p. 90-106.

IAPAR. Cartas climáticas do Paraná. Disponível em:

http://www.iapar.br/modules/conteudo/conteudo.php?conteudo=863. 1998. Acesso: 18 nov. 2015.

IBGE. Ministério do Planejamento e Orçamento. Mapa de vegetação do Brasil. Brasília, DF, 1993. 1 mapa, color., $120 \mathrm{~cm}$ x 0,80 cm. Escala 1: 5000.000 .

ITCG, 2009. Formações Fitogeográficas - Estado do Paraná. Disponível em: http://www.itcg.pr.gov.br/arquivos/File/Produtos_DGEO/Mapas_ITCG/PDF/Mapa_Fitogeogr afico_A3.pdf. Acesso: 17 nov. 2015.

LOUZADA, F. L. R. O.; SANTOS, A.R.; SILVA, A. G. Delimitação de corredores ecológicos no arcgis 9.3. Alegre: Caufes, 2010.

MCGARIGAL, K. \& MARKS, B.J. Fragstats: Spatial Analysis Program for Quantifying Landscape Structure. USDA Forest Service General Technical Report PNW-GTR-351. 1994.

McGARIGAL, K., S.A. CUSHMAN \& ENE, E. FRAGSTATS: Spatial Pattern Analysis Program for Categorical and Continuous Maps. Computer software program produced by the authors at the University of Massachusetts. Disponível em:

http://www.umass.edu/landeco/research/fragstats/fragstats.html. 2012. 

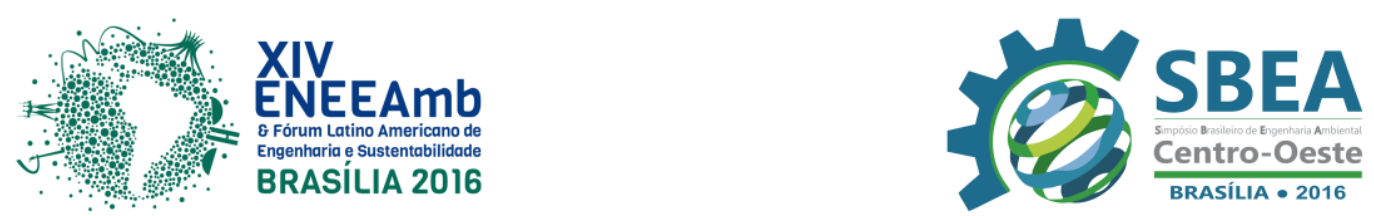

MINEROPAR, 2006. Mapa Geomorfológico do Estado do Paraná. Disponível em:

http://www.mineropar.pr.gov.br/arquivos/File/2_Geral/Geomorfologia/Atlas_Geomorforlogic o_Parana_2006.pdf. Acesso: 15 nov. 2015.

MINEROPAR,2001. Mapa Geológico do Paraná. Disponível em:

http://www.mineropar.pr.gov.br/arquivos/File/MapasPDF/atlasgeo.pdf. Acesso:17 nov. 2015.

ROBBINS C.; DAWSON, D. \& DOWELL, B. Habitat area requirements of breeding forest birds of the Middle Atlantic States. Wildl. Monogr. 1989. p. 34.

SEMA. Bacias Hidrográficas do Paraná. Disponível em:

http://www.meioambiente.pr.gov.br/arquivos/File/corh/Revista_Bacias_Hidrograficas_do_Par ana.pdf . 2010. Acesso: 15 nov. 2015.

SOUZA, B.; COSTA, R.I.R.; LOUZADA, J.N.C. Influência do tamanho e da forma de fragmentos florestais na composição da taxocenose de crisopídeos (Neuroptera:

Chrysopidae). Arq. Inst. Biol., São Paulo, v.75, n.3, p.351-358, jul./set., 2008. Disponível em: http://www.biologico.sp.gov.br/docs/arq/v75_3/souza1.pdf.

VELOSO, H. P.; FILHO, A. L. R. R.; LIMA, J. C. A. Classificação da vegetação brasileira, adaptada a um sistema universal. IBGE. Rio de Janeiro, RJ. 1991. p. 124. 